\title{
PROCESSAMENTO AUDITIVO \\ DA INFORMAÇÃO EM SUJEITOS COM AFASIA
}

\author{
Jordana da Silva Folgearini \\ Eliara Pinto Vieira Biaggio² \\ Rúbia Soares Bruno ${ }^{3}$ \\ Sheila Jacques Oppitz ${ }^{4}$ \\ Elenir Fedosse ${ }^{5}$ \\ Sinéia Neujahr dos Santos ${ }^{6}$ \\ Michele Vargas Garcia?
}

resumo

Introdução: Diferentes tipos de alteração no sistema nervoso central (SNC) podem resultar em diversos tipos de distúrbios de linguagem e/ou fala. Havendo alguma alteração em nível central, é possível que

1 Graduação em Fonoaudiologia. Mestranda em Distúrbios da Comunicação Humana. E-mail: johfolgearini@hotmail.com.

2 Graduação em Fonoaudiologia. Doutorado em Distúrbios da Comunicação Humana (Fonoaudiologia). Professor Adjunto da Universidade Federal de Santa Maria. E-mail: eliarapv@yahoo.com.br.

3 Graduação em Fonoaudiologia. Mestranda em Distúrbios da Comunicação Humana. E-mail: rubia_bee@hotmail.com

4 Graduação em Fonoaudiologia. Doutoranda em Distúrbios da Comunicação Humana. E-mail: she_oppitz@hotmail.com.

5 Graduação em Fonoaudiologia. Doutorado em Linguística. Professora do curso de Fonoaudiologia da Universidade Federal de Santa Maria. E-mail: efedosse@gmail.com.

6 Graduação em Fonoaudiologia. Doutoranda em Distúrbios da Comunicação Humana. E-mail: sineians@gmail.com.

7 Graduação em Fonoaudiologia. Doutorado em Ciências pela UNIFESP. Professora Adjunta do curso de Fonoaudiologia e do Programa de Pós-Graduação em Distúrbios da Comunicação Humana da Universidade Federal de Santa Maria. E-mail: michelemvg@outlook.com. 
algumas habilidades auditivas estejam alteradas. Objetivo: Avaliar as habilidades do processamento auditivo de modo comportamental e eletrofisiológico em sujeitos com afasia de expressão e identificar a correlação entre as avaliações. Métodos: Participaram do estudo sujeitos com idade entre 29 e 72 anos, com afasia de expressão decorrente de lesão encefálica (acidente vascular cerebral ou traumatismo crânio encefálico). Esses indivíduos foram submetidos a uma avaliação audiológica básica, além da avaliação comportamental e eletrofisiológica do processamento auditivo, que contemplou os seguintes testes: localização sonora, memória sequencial para sons verbais e não verbais, padrão de frequência e duração, dicótico de dígitos, fala comprimida, identificação de sentenças sintéticas e dicótico não verbal, além do Potencial Evocado Auditivo de Longa Latência (PEALL) P300. Resultados: Todos os sujeitos avaliados apresentaram alteração na maioria das habilidades comportamentais de processamento auditivo. No PEALL, todos apresentaram os potenciais exógenos (N1, P2) dentro da latência esperada. Apenas um não apresentou o potencial P300 bilateralmente. Os outros cinco afásicos apresentaram P300 bilateralmente. Conclusão: Foi possível avaliar as habilidades do processamento auditivo em quase todos os sujeitos com afasia de expressão de modo comportamental e eletrofisiológico. Não foi observada correlação significativa entre as avaliações, identificando que as avaliações comportamentais foram piores do que as eletrofisiológicas.

palavras-chave

Afasia. Percepção Auditiva. Audição. Audiologia. Potenciais Evocados.

\section{Introdução}

A linguagem, seja falada ou escrita, desempenha um papel fundamental em todas as atividades que realizamos. Ela não é um simples veículo de informação, mas o resgate do homem como um ser social, histórico e cultural. A linguagem promove a (re)organização contínua da história de cada sujeito, tornando-os autores da vida singular, que está em constituição permanente, e a interação social depende de processos interlocutivos que se efetivam no espaço de produção da linguagem (MASSI et al., 2010). A linguagem é dividida em subsistemas localizados em regiões específicas do cérebro, o qual não age separadamente, mas como um todo.

Diferentes tipos de alteração no sistema nervoso central (SNC) podem resultar em diversos tipos de distúrbios de linguagem e/ou fala. Esses distúrbios 
possuem diversas causas, e entre as mais comuns que podem levar a alteração na comunicação humana estão o trauma crânio encefálico (TCE) e o acidente vascular cerebral (AVC), tanto hemorrágico como isquêmico, que são fatores etiológicos frequentemente associados aos distúrbios de linguagem. Nesse contexto, as afasias são tradicionalmente definidas como um transtorno adquirido da linguagem causado por lesão encefálica, que pode estar associado a outras alterações cognitivas, interferindo na capacidade de processamento e/ ou produções linguísticas. Trata-se de uma das alterações neurológicas mais comuns após lesão focal adquirida no sistema nervoso central, em áreas responsáveis pela linguagem compreensiva e/ou expressiva, oral e/ou escrita (TALARICO; VENEGAS; ORTIZ, 2011).

Dessa forma, havendo alguma alteração em nível central, é possível que algumas habilidades auditivas estejam alteradas. O processamento auditivo refere-se àquilo que se faz com o que se ouve, e envolve habilidades como: resolução temporal, ordenação temporal, fechamento auditivo, síntese binaural, figura-fundo, localização sonora, atenção e memória (ASHA, 2005).

Não basta, portanto, possuir limiares auditivos normais: é preciso que o sinal acústico seja analisado e interpretado para que se transforme em uma mensagem com significado. Para isso, é necessária integridade orgânica e funcional de todo sistema auditivo (SANTOS et al., 2013). Para avaliar as habilidades comportamentais do processamento auditivo, tem-se disponível uma bateria de testes comportamentais (PEREIRA; SCHOCHAT, 2011). Esses testes são escolhidos de acordo com a capacidade do sujeito em realizar as tarefas exigidas em cada teste. Dentre eles, está o teste dicótico de dígitos, de fácil execução, que avalia a habilidade de figura-fundo para sons verbais, podendo ser aplicado na etapa de integração e separação binaural e também sendo um teste muito útil na avaliação do sujeito com afasia.

De igual importância na bateria que avalia os sujeitos afásicos, está o Potencial Evocado Auditivo de Longa Latência (PEALL). Esse teste eletrofisiológico é um método objetivo, utilizado para avaliar a via auditiva central (do nervo auditivo até o córtex cerebral) em resposta a um estímulo acústico. O potencial cognitivo P300 é um potencial endógeno, que surge 300 ms após a estimulação acústica e sua geração envolve os processos de atenção, discriminação auditiva e memória (SUTTON et al., 1965). Em afásicos, a realização desse potencial permite a identificação fisiológica da função auditiva central, com foco em memória auditiva.

Tanto a avaliação comportamental do processamento auditivo como a avaliação eletrofisiológica trazem contribuições importantes para se pensar nas possibilidades de terapia fonoaudiológica para o sujeito afásico, uma vez 
que reúne diversos aspectos da constituição do sujeito no processo de reabilitação, envolvendo a linguagem e as habilidades do processamento auditivo.

Assim, o objetivo deste estudo é avaliar as habilidades do processamento auditivo de modo comportamental e eletrofisiológico em sujeitos com afasia de expressão e identificar a correlação entre as avaliações.

\section{Métodos}

Este estudo apresentou caráter descritivo, quantitativo e transversal. $\mathrm{O}$ projeto de pesquisa foi aprovado pelo comitê de ética desta instituição, sobre o protocolo de número 25933514.1.0000.5346. Todos os sujeitos assinaram o Termo de Consentimento Livre e Esclarecido (TCLE). Seguindo os princípios éticos em pesquisa, os sujeitos foram beneficiados com as avaliações básicas e complementares da função auditiva.

A amostra foi constituída de sujeitos afásicos (afasia de expressão) do Grupo Interdisciplinar de Convivência de Sujeitos com Lesão Encefálica (GICSLE), pertencente a uma universidade no interior do estado do Rio Grande do Sul. Nesse grupo, são realizados encontros semanais com atividades terapêuticas, buscando a troca ativa e intensa entre os participantes para que estes saiam do isolamento social que a afasia proporciona. As atividades são voltadas para o trabalho em equipe e a interação entre os sujeitos. Além das atividades em grupo, todos os envolvidos recebem, também, terapia fonoaudiológica semanal individual.

Fazem parte do GICSLE doze sujeitos com idade entre 29 e 72 anos, com média de idade de 58 anos, e todos aceitaram participar das avaliações audiológicas. O grupo estudado, com exceção de uma afásica que sofreu um traumatismo craniano, apresentou a sequela por acidente vascular cerebral. $\mathrm{O}$ transtorno (afasia de expressão) foi diagnosticado por médico neurologista. Ao serem convidados para as avaliações, os sujeitos foram informados da possibilidade de treinamento auditivo após as avaliações no caso das mesmas apresentassem alteração, sendo realizadas na clínica escola da mesma instituição da pesquisa.

Foram incluídos no estudo os sujeitos com afasia de expressão, com audição normal em ambas as orelhas (LLOYD; KAPLAN, 1978). Dessa forma, foram excluídos dois sujeitos com afasia de compreensão associada à afasia de expressão, impossibilitando a realização de todos os procedimentos. Sujeitos com alteração de orelha média também foram excluídos do estudo. A coleta foi realizada em um Hospital Universitário no interior do Rio Grande do Sul (RS).

A avaliação contemplou anamnese, inspeção visual do meato acústico externo (MAE), Audiometria Tonal Liminar (ATL), testes de fala, Medidas de 
Imitância Acústica (MIA) e aspectos do processamento auditivo (PA) referentes às habilidades de localização sonora, memória sequencial para sons verbais e não verbais, resolução e ordenação temporal e figura-fundo para sons verbais (integração e separação binaural), fechamento auditivo, figura-fundo para sons verbais com associação entre estímulos auditivos e visuais e figura-fundo para sons não verbais.

Para avaliar tais habilidades, os testes foram escolhidos, buscando maior compreensão do sujeito, sendo eles o de localização sonora, memória sequencial para sons verbais e não verbais, padrão de frequência e duração, dicótico de dígitos, fala comprimida, identificação de sentenças sintéticas e dicótico não verbal. Todos os testes comportamentais foram aplicados a $40 \mathrm{dBNS}$, levando em consideração a sensação de conforto, utilizando o Audiômetro Itera II, com fones TDH 39 acoplados a um CD player.

A avaliação eletrofisiológica foi realizada por meio do Potencial Evocado Auditivo de Longa Latência (PEALL) P300 utilizando o equipamento Intelligent Hearing System (IHS), a 70-80dB, buscando conforto e audibilidade dos sujeitos.

Para uma melhor compreensão do desenho metodológico, segue o organograma abaixo:

Figura 1 - Organograma com a distribuição do desenho metodológico.

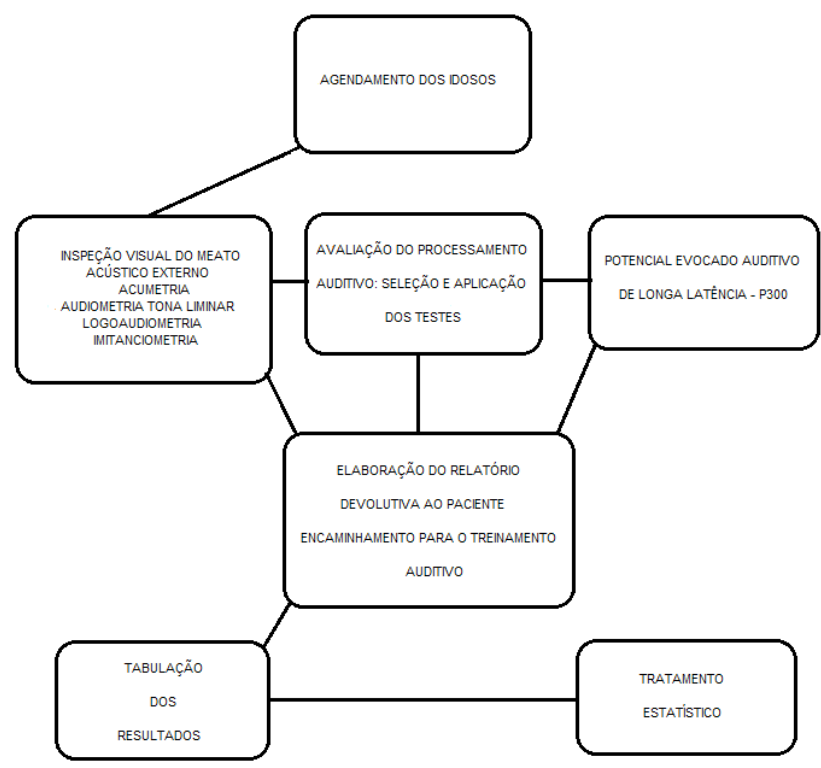

Fonte: Figura elaborada pelos autores. 
Os procedimentos realizados foram os seguintes:

(1) Anamnese, utilizando um protocolo com perguntas sobre dados de identificação (nome, sexo, idade, data de nascimento, profissão, escolaridade), queixa principal, histórico audiológico (otalgia, otorreia, otites, zumbido, tontura), doenças, uso de medicação, antecedentes familiares com perda auditiva, fumo, álcool, exposição a ruído (quantas horas, por quanto tempo, se faz uso de protetor auricular), uso de dispositivos sonoros (MP3, Ipod), dificuldade de falar em ambiente ruidoso e dificuldade de falar ao telefone.

(2) Inspeção visual do meato acústico externo por meio de otoscópio.

(3) Avaliação audiológica por meio da Audiometria Tonal Liminar (ATL) com audiômetro de 2 canais (Itera II), com fones (TDH 39). Na ATL, foram pesquisados os limiares de via aérea nas frequências de $250 \mathrm{~Hz}, 500 \mathrm{~Hz}, 1000$ $\mathrm{Hz}, 2000 \mathrm{~Hz}, 3000 \mathrm{~Hz}, 4000 \mathrm{~Hz}, 6000 \mathrm{~Hz}$ e $8000 \mathrm{~Hz}$. A técnica utilizada é a descendente-ascendente. Foram considerados indivíduos normo-ouvintes aqueles que apresentaram média tritonal $(500 \mathrm{~Hz}, 1000 \mathrm{~Hz}$ e $2000 \mathrm{~Hz})$ menor ou igual a 25 dBNA (decibel Nível de Audição) (LLOYD; KAPLAN, 1978).

(4) Logoaudiometria, pesquisando o limiar de reconhecimento de fala (LRF), utilizando uma lista de palavras dissílabas, e do índice percentual de reconhecimento de fala (IPRF), utilizando uma lista de palavras monossílabas. Tanto o LRF como o IPRF foram realizados sem cabine acusticamente tratada.

(5) As Medidas de Imitância Acústica (MIA) foram realizadas pelo analisador de orelha média da marca Interacoustics Modelo AT 235 e tom-sonda $226 \mathrm{~Hz}$, para pesquisa da curva timpanométrica e dos reflexos acústicos. Estes foram pesquisados nas frequências de $500 \mathrm{~Hz}$ a $4000 \mathrm{~Hz}$ bilateralmente, no modo contralateral (HALL III; CHANDLER, 1999).

(6) Os testes comportamentais para a avaliação do processamento auditivo foram realizados a $40 \mathrm{dBNS}$ (a partir da média das frequências de $500 \mathrm{~Hz}, 1000$ $\mathrm{Hz}$ e $2000 \mathrm{~Hz}$ ), então garantindo a audibilidade e o conforto do paciente. Todos os testes foram transformados em porcentagem para padronizar a tabela de resultados e facilitar a análise.

(6. 1) O teste de localização sonora (LS) foi realizado em campo livre, onde os indivíduos foram orientados a fechar os olhos e localizar a fonte sonora em cinco direções (direita, esquerda, atrás, acima e à frente). Esse teste visa a buscar informações sobre a interação binaural e a habilidade auditiva avaliada é a de localização sonora.

(6. 2) Teste de Memória Sequencial para sons verbais e não verbais (MSSV/ MSSNV): a tarefa é ordenar sons em uma sequência temporal ou sílabas simples e sons de objetos sonoros de espectro muito diferentes entre si. 
(6. 3) Teste Padrão de Frequência (TPF) e Teste Padrão de Duração (TPD): os testes de reconhecimento de padrão de frequência e duração avaliam a habilidade auditiva de ordenação temporal. A função medida é a de discriminação de padrões sonoros, ou seja, se os sons eram graves ou agudos, ou se eram curtos ou longos.

(6. 4) Teste Dicótico de Dígitos (TDD): o teste apresenta dois dígitos em cada orelha simultaneamente, e, no sujeito, foram aplicadas duas tarefas, a de integração binaural (em que o indivíduo tinha de repetir oralmente os quatro dígitos apresentados em ambas as orelhas) e a tarefa de separação binaural/ escuta direcionada (em que tinha de repetir oralmente dois dígitos da orelha solicitada, direita ou esquerda). Foi solicitado ao sujeito reproduzir os estímulos apresentados independentemente da ordem de apresentação dos mesmos. $\mathrm{O}$ TDD possibilita avaliar a habilidade auditiva de figura-fundo para sons verbais.

(6. 5) Fala Comprimida (FC): exige que o sujeito repita oralmente as listas com cinquenta palavras (dissílabas) de cada orelha solicitada com compressão de $60 \%$. O teste avalia a habilidade de fechamento auditivo.

(6. 6) Teste de Identificação de Sentenças Sintéticas (SSI): avalia a habilidade de figura fundo para sons verbais, sendo que, nesta pesquisa, foi realizada apenas a etapa dicótica. O sujeito foi instruído a apontar a frase ouvida e ignorar a presença da mensagem competitiva. A frase foi apresentada em uma orelha e a mensagem competitiva na orelha contralateral - realizadas nas relações sinal-ruído 0 e -40, com dez estímulos (frases) em cada relação. A relação -40 só foi aplicada quando o sujeito apresentou normalidade na relação 0 .

(6. 7) Teste Dicótico Não Verbal (TDNV): permite analisar o processamento dicótico com sons não linguísticos em escuta dicótica e separação binaural. Prevê uma resposta motora de apontar as figuras que representam os estímulos sonoros.

(6. 8) O PEALL foi pesquisado em ambiente silencioso, sendo que o indivíduo teve de permanecer em estado de alerta e contar os estímulos raros $(20 \%$ do total de estímulos) que aparecerão aleatoriamente e ignorar os estímulos frequentes ( $80 \%$ do total de estímulos). Os eletrodos serão fixados à pele do indivíduo com pasta condutiva eletrolítica no vertex $(\mathrm{Cz})$, mastóide esquerda (A1), mastóide direita (A2) e o terra na testa. O equipamento utilizado foi o modelo SmartEP da marca Intelligent Hearing Systems (IHS). A impedância dos eletrodos foi inferior a $3 \mathrm{Kohm}$. Foi apresentada uma série de 300 estímulos (240 frequentes e 60 raros) com estímulos verbais (sílabas /ba/, estímulo frequente, e /di/, estímulo raro) a uma intensidade de $75 \mathrm{~dB}$ NA. Os valores de latência e amplitude foram obtidos pela identificação das ondas N1, P2, e P300, esperadas, respectivamente, com N1 entre $80 \mathrm{~ms}$ a $150 \mathrm{~ms}$, P2 entre $145 \mathrm{~ms}$ a $180 \mathrm{~ms}$ e P3 
entre 220 a 380 ms, segundo classificação de McPherson (1996), e a amplitude mínima de P3 de $3 \mu \mathrm{V}$, segundo classificação de Oliveira, Murphy e Schochat (2013). Para a marcação do componente P300, considerou-se o primeiro pico positivo no traçado dos estímulos raros, comparando os traçados dos exógenos com o endógeno, o P300 só foi marcado após o aparecimento do complexo exógeno (MCPHERSON, 1996).

Após o levantamento dos dados, foi realizada uma análise descritiva do desempenho dos indivíduos. A fim de verificar se há correlação estatística entre os testes comportamentais e os eletrofisiológicos, foi utilizado o teste de correlação de Sperman, em virtude dos dados não apresentarem distribuição normal. O nível de significância adotado foi de $\mathrm{p} \leq 0,05$.

\section{Resultados}

Foram agendados para avaliação completa dez sujeitos, pois dois apresentavam afasia de compreensão associada à afasia de expressão, impossibilitando a realização dos procedimentos do estudo. Destes, seis conseguiram finalizar todos os procedimentos, sendo que os outros quatro precisaram ser excluídos por não conseguirem realizar os testes. Os sujeitos de 1 a 4 conseguiram realizar todos os mesmos testes, e os sujeitos 5 e 6 apresentavam fala ininteligível, sendo necessário trocar o teste dicótico (TDD por TDNV).

Os dados de MIA não são apresentados, pois foram usados apenas para atender os critérios de elegibilidade do estudo, sendo que nenhum sujeito foi excluído por alterações de orelha média.

Na Tabela 1, seguem os achados comportamentais, na Tabela 2, os achados eletrofisiológicos, e no Quadro 1, a correlação entre os mesmos. 


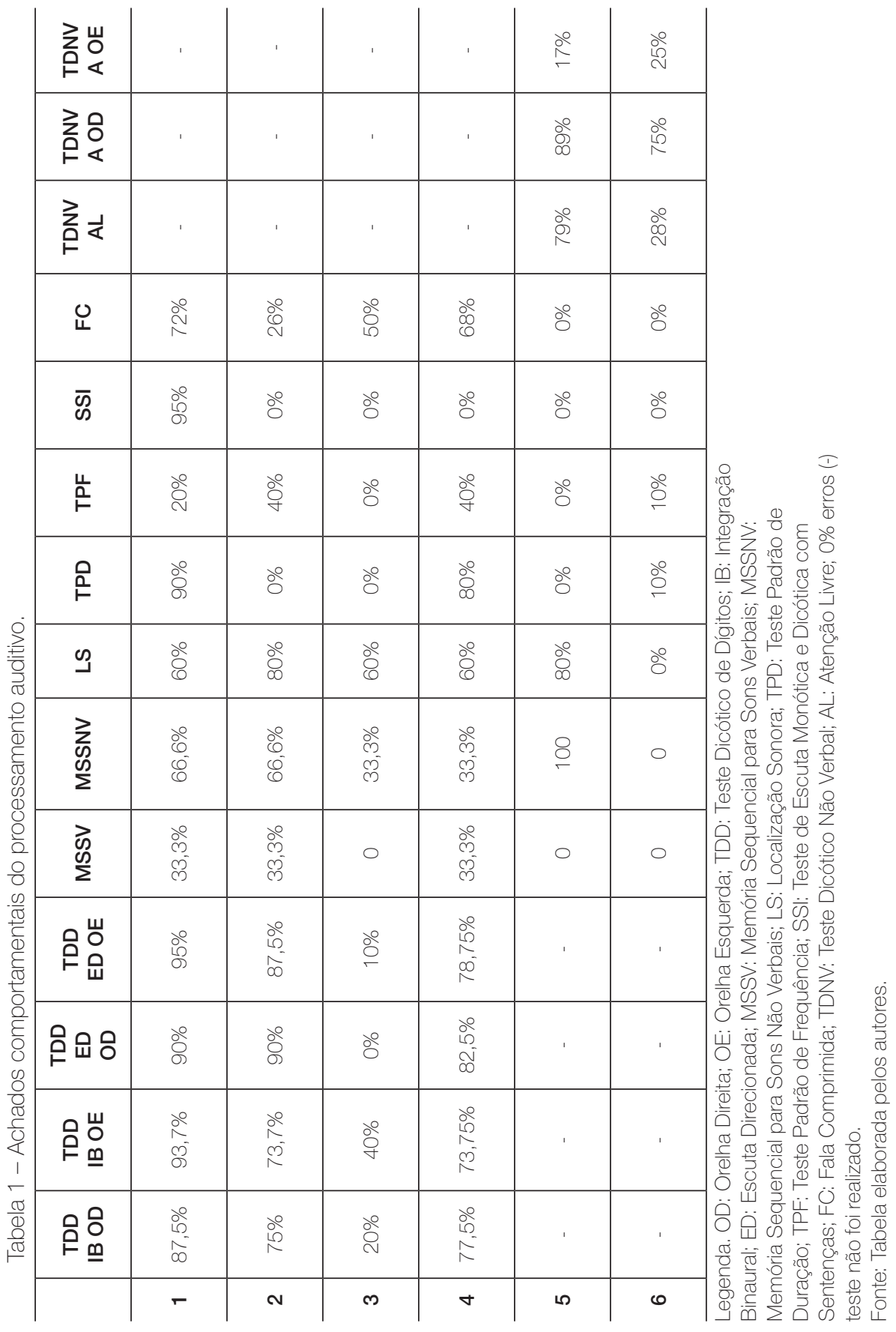




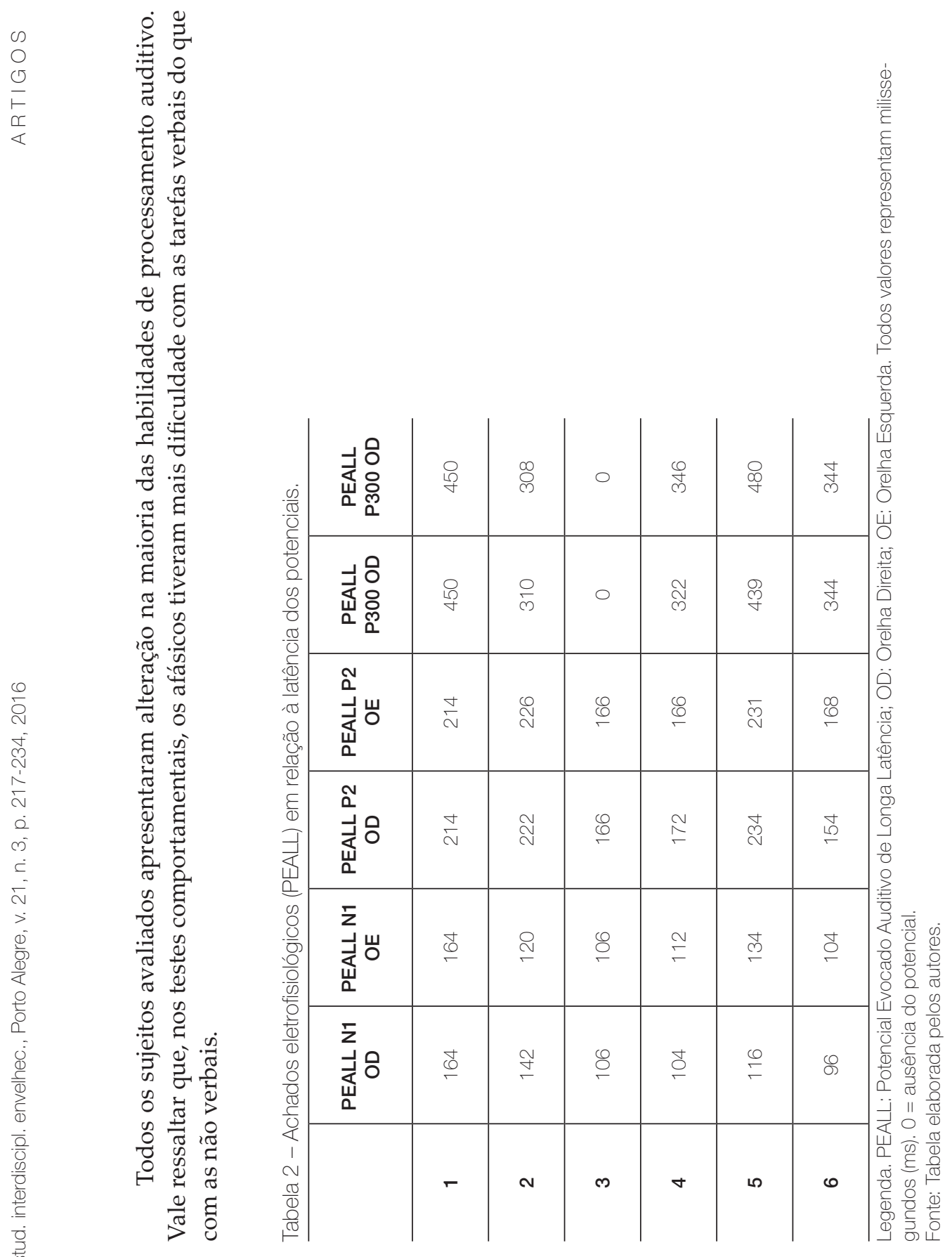


No PEALL, todos apresentaram os potenciais exógenos (N1, P2) dentro da latência esperada. Apenas um não apresentou o potencial P300 bilateralmente, sendo que os outros cinco afásicos apresentaram.

Quadro 1 - Correlação entre os testes comportamentais e eletrofisiológicos.

\begin{tabular}{|c|l|l|l|l|l|l|}
\hline & N1 OD & \multicolumn{1}{|c|}{ P2 OD } & P300 OD & \multicolumn{1}{|c|}{ N1 OE } & \multicolumn{1}{|c|}{ P2 OE } & P300 OE \\
\hline DDIBOD & 0,658374 & 0,826848 & 0,492571 & - & - & - \\
\hline DDIBOE & - & - & - & 0,774057 & 0,165057 & 0,863763 \\
\hline DDEDOD & 0,268998 & 0,218894 & 0,804819 & - & - & - \\
\hline DDEDOE & - & - & - & $0,037386^{*}$ & 0,218894 & 0,747060 \\
\hline MSSV & 0,326164 & 0,573392 & 1,000000 & 0,326164 & 1,000000 & 0,854080 \\
\hline MSSNV & 0,059028 & $0,001249^{*}$ & 0,525115 & $0,019820^{*}$ & 0,052772 & 0,248771 \\
\hline LOC SON & 0,191744 & $0,008050^{*}$ & 0,790048 & 0,191744 & 0,130162 & 0,681004 \\
\hline TPD & 0,068727 & 0,267778 & 0,948571 & 0,267778 & 0,241716 & 0,949310 \\
\hline TPF & 0,551786 & 0,861524 & 0,329171 & 0,551786 & 0,744236 & 0,594094 \\
\hline SSIOD & 0,158302 & 0,804726 & 0,150200 & - & - & - \\
\hline SSIOE & - & - & - & 0,158302 & 0,801909 & 0,441113 \\
\hline FCOMPOD & 0,424662 & 0,913132 & 0,867991 & - & - & - \\
\hline FCOMPOE & - & - & - & 0,571370 & 0,287631 & 0,955876 \\
\hline TDNVAL & 0,158302 & 0,158302 & 0,801909 & 0,158302 & 0,801909 & 0,804726 \\
\hline TDNVOD & 0,414430 & 1,000000 & 0,406940 & - & - & - \\
\hline TDNVOE & - & - & - & 0,693907 & 0,406940 & 0,414430 \\
\hline P & & & & \\
\hline
\end{tabular}

${ }^{\star}$ p significante < 0,05, para o teste de correlação de Spearman.

Legenda. DDIBOD - Dicótico de Dígitos, Integração Binaural, Orelha Direita - DDIBOE: Dicótico de Dígitos, Integração Binaural, Orelha Esquerda - DDEDOD: Dicótico de Dígitos, Escuta Direcionada, Orelha Direita - DDEDOE: Dicótico de Dígitos, Escuta Direcionada, Orelha Esquerda - MSSV - Memória Sequencial para Sons Verbais - MSSNV: Memória Sequencial para Sons não Verbais - LOC SON: Localização Sonora - TPD: Teste Padrão de Duração - TPF: Teste Padrão de Frequência- SSIOD: Teste de Identificações de Sentenças com Mensagem Competitiva, Orelha Direita - SSIOE: Teste de Identificações de Sentenças com Mensagem Competitiva, Orelha Esquerda- FCOMPOD: Fala Comprimida, Orelha Direita - FCOMPOE: Fala Comprimida, Orelha Esquerda - TDNVAL: Teste Dicótico Não Verbal, Atenção Livre: TDNVOD - Teste Dicótico Não Verbal, Orelha Direita - TDNVOE - Teste Dicótico Não Verbal, Orelha Esquerda.

Fonte: Quadro elaborado pelos autores. 
Ao realizar a correlação entre os achados comportamentais e eletrofisiológicos, foi observado que o grupo de afásicos apresenta piores resultados na avaliação comportamental do que na avaliação dos Potenciais Evocados Auditivos de Longa Latência (Quadro 1). Assim, não houve correlação entre os testes nessa população, indicando que os afásicos apresentam maior dificuldade comportamental do que restrição no funcionamento eletrofisiológico da via auditiva em nível cortical.

\section{Discussão}

Avaliar o processamento auditivo nessa população, tanto comportamental como eletrofisiologicamente, ainda não faz parte da rotina clínica fonoaudiológica. Porém, as informações que essas avaliações trazem para o processo terapêutico e para prognóstico da reabilitação são muito enriquecedoras para o fonoaudiólogo. Com essas informações, o profissional pode orientar melhor a família quanto às possibilidades de evolução e expectativas em relação ao caso. Essa afirmativa vai ao encontro do citado pelos autores (ALVARENGA et al., 2005b). Na prática clínica, a avaliação fonoaudiológica do indivíduo afásico requer atenção do profissional responsável, pois depende de inúmeras variáveis, dentre as quais se ressalta a dificuldade de respostas consistentes em razão da sequela neurológica e a existência de comprometimentos motores associados que alteram a comunicação de modo geral. Dessa maneira, é de extrema importância uma cuidadosa análise do examinador quanto aos processos receptivos, referentes tanto à percepção do som como ao processamento das informações auditivas até centros corticais. A dificuldade de expressão deste paciente pode implicar um viés na interpretação dos dados da avaliação quanto às habilidades auditivas e, consequentemente, na determinação do diagnóstico fonoaudiológico preciso. A amostra deste estudo não pôde ser maior, pois, na instituição na qual a pesquisa foi realizada, esse é o número de afásicos em tratamento fonoaudiológico e também de participantes do grupo.

Um estudo com pacientes epilépticos com lesões cerebrais utilizou estímulo verbal em tarefas de escuta dicótica. O objetivo do trabalho foi investigar o reconhecimento de sons verbais em indivíduos com dominância hemisférica esquerda e em indivíduos com dominância hemisférica direita. Os resultados mostraram que a orelha contralateral ao hemisfério dominante para estímulos de fala é mais eficiente no reconhecimento de sons verbais. Esse efeito foi independente da preferência manual e do local da descarga elétrica. Isso confirma que a via contralateral é preferencial em relação à via ipsilateral e 
que o lobo temporal dominante é mais importante do que o lobo temporal não dominante na percepção de estímulos de fala (KIMURA, 2001). A presente pesquisa encontrou resultados que não vão ao encontro desse estudo, tendo em vista que não houve melhores resultados na orelha do hemisfério dominante.

Pesquisadores avaliaram a habilidade em identificar padrões de sons que diferiam quanto à frequência sonora e quanto à intensidade em três sujeitos que foram submetidos à secção cirúrgica do corpo caloso. Os indivíduos apresentavam limiares auditivos normais e preferência manual direita. Um dos indivíduos foi avaliado antes da secção cirúrgica do corpo caloso dez dias após o procedimento cirúrgico e um ano após o procedimento cirúrgico. Os resultados indicaram que a seç̧ão cirúrgica do corpo caloso afetou a habilidade em nomear verbalmente o padrão tonal, no teste de padrão de frequência e no teste de padrão de intensidade. No entanto, não foram observadas dificuldades quando a resposta exigida era imitar (humming) as sequências ouvidas no teste de padrão de frequência. Sendo assim, os autores concluíram que nomear o padrão tonal implicava transferência de informação acústica via corpo caloso, enquanto que o mesmo não ocorria quando a resposta exigida era de humming (MUSIEK; PINHEIRO; WILSON, 1980). Por buscar maior nível de exigência na avaliação dos indivíduos com lesão encefálica, o presente estudo foi ao encontro do estudo supracitado e os sujeitos responderam ao teste TPD e TPF com nomeação, da mesma forma que, no estudo referido anteriormente, encontramos alterações nos achados.

A ênfase no fato de as alterações de processamento auditivo estarem relacionadas especificamente à modalidade auditiva tem levado muitos autores a concluir que o diagnóstico de alteração do processamento auditivo somente pode ser dado quando há um défice que é específico da modalidade auditiva e de nenhuma outra modalidade. Certamente, é razoável insistir que um défice auditivo específico seja demonstrado antes de aplicar o rótulo de desordem do processamento auditivo (DPA). No entanto, esperar que um défice específico da modalidade auditiva existirá, exclusivamente, em todos os casos de DPA, excluindo-se défices em outras modalidades sensoriais, parece ser neurofisiologicamente insustentável sob a luz dos conhecimentos atuais e da alta complexidade do sistema nervoso auditivo central (SNAC) (BELLIS, 2011). Nosso estudo concorda com a autora referida, pois encontramos alterações nas habilidades de processamento auditivo em sujeitos com importante prejuízo na linguagem expressiva.

Vinte afásicos foram estudados utilizando o teste de identificação de sentenças Pediatric Speech Inteligibility (PSI), versão em português, e encontram alteração nos achados (ORTIZ; PERONI, 2008). Nossa pesquisa concorda 
com o estudo supracitado em relação à escolha do teste utilizado para avaliar tal população e ainda em relação à alteração nas avaliações.

Ortiz e Peroni (2008) realizaram o mesmo teste na mesma população e observaram dificuldades de compreensão estatisticamente significantes na situação de mensagem competitiva ipsilateral, nas situações $0 \mathrm{~dB}$ e $-10 \mathrm{~dB}$, além de dificuldades de compreensão também na situação de mensagem competitiva contralateral MCC (- $40 \mathrm{~dB})$. Os achados desse estudo concordam com a pesquisa anteriormente realizada.

Jerger (1987) realizou um estudo em indivíduos com lesão cerebral adquirida, e o teste de logoaudiometria pediátrica foi aplicado em crianças e a situação de escuta dicótica mostrou-se um bom indicador de alterações do tipo lesional em nível de lobo temporal. Nesse estudo, os pacientes afásicos apresentavam lesões cerebrais de hemisfério esquerdo, não necessariamente na área temporal. Porém, é interessante verificar que, em tal condição, os pacientes afásicos tiveram desempenho similarmente ruim em ambas as orelhas, apesar de apresentarem lesão apenas em hemisfério esquerdo. O mau desempenho em ambas as orelhas pode ser explicado pela complexidade do processamento da informação e do seu padrão de resposta. A audição é um processo complexo que orquestra a transdução do estímulo acústico para impulsos neurais, transmissão dos impulsos neurais pelo nervo auditivo, a transmissão destes ao cérebro e, consequentemente, a elaboração cognitiva e o registro perceptual do sinal acústico pelo cérebro, sendo que apenas a integridade de todo o sistema auditivo pode promover o processamento adequado da informação auditiva (HUSAIN; HORWITZ, 2006).

Dias (2005) realizou um estudo envolvendo linguagem, processamento auditivo e síndrome de Asperger e observou que indivíduos com tal síndrome apresentaram alterações no processamento auditivo e que essas alterações se associaram às modificações de linguagem. Os testes comportamentais de processamento utilizados foram TDD, TPD e TPF. O presente estudo vai ao encontro da autora referida, concordando que é necessário realizar avaliação do processamento auditivo em sujeitos com alteração de linguagem e, ainda, a escolha dos testes utilizados foi a mesma.

O registro normal do potencial cognitivo $\mathrm{P} 300$ em indivíduos afásicos já foi descrito anteriormente por Alvarenga et al. (2005a), indo o presente estudo ao encontro desses achados. Esse mesmo estudo sugere que o aumento da latência do P300 encontrado nos indivíduos afásicos pode ser justificado pela sequela da lesão neurológica, agravada por comportamentos inerentes ao quadro clínico como a fatigabilidade, a qual os indivíduos afásicos tendem a apresentar em situações que exigem atenção concentrada. Semelhante raciocínio pode ser feito 
para os indivíduos que apresentaram ausência do P300. Cabe lembrar que o aparecimento do componente P300 está relacionado aos processos cognitivos de atenção, discriminação auditiva e memória. Na presença de lesão cerebral, tais processos descritos podem estar comprometidos, o que poderia explicar o aumento da latência e redução da amplitude ou ausência do P300 nos indivíduos afásicos, como observado na presente pesquisa.

Alvarenga et al. (2005a), em um estudo com oito afásico, observaram aumento da latência do P300, o que pode ser justificado pela sequela da lesão neurológica, agravada por comportamentos inerentes ao quadro clínico como a fatigabilidade, na qual os indivíduos afásicos tendem a apresentar fadiga em situações que exigem atenção concentrada. Nosso estudo vai ao encontro do supracitado, pois a metade dos afásicos avaliados (Tabela 2) apresentaram latência aumentada (2 deles) ou não apresentaram a onda (1). Cabe lembrar que o aparecimento do componente $\mathrm{P} 300$ está relacionado aos processos cognitivos refletidos nos componentes e subcomponentes das habilidades de atenção, discriminação auditiva e memória (PICTON; HILLYARD, 1974).

Outro estudo observou a integridade das vias auditivas em pacientes portadores de Doença de Parkinson (DP) (PINEROLI et al., 2002). Uma vez que a latência do P300 aumenta de forma linear com a idade, a partir dos 45 anos, aceitando-se um aumento de $1 \mathrm{~ms}$ a 1,5 ms por ano, observou-se integridade nas vias cerebrais que refletem a habilidade de desempenho cognitvo em pacientes portadores de DP, sem comprometimento demencial exuberante. $\mathrm{O}$ mesmo fato pode ser observado no presente estudo nos sujeitos com afasia, em que o aumento de latência pode ser pela idade dos afásicos, sendo a média de idade de 58 anos.

Melo et al. (2007), comparando o PEALL com a avaliação de linguagem, observou alterações nos achados do PEALL no caso descrito. Nosso estudo não vai ao encontro de tais achados, visto que a maioria dos afásicos apresentou normalidade nos achados do PEALL. Esse fato pode ser justificado pela diferença no tempo de lesão, visto que os sujeitos do nosso grupo apresentam lesão há mais tempo (mais de 20 meses), já podendo ter sofrido os efeitos da plasticidade e reorganização cortical.

Marangoni et al. (2013) compararam o Potencial Evocado Auditivo de Tronco Encefálico (PEATE) e o PEALL de pacientes que sofreram Trauma Crânio Encefálico (TCE) com os de indivíduos normais, e observaram aumento da latência e diminuição da amplitude nos pacientes que tinham sofrido TCE com relação aos normais, porém sem significância estatística. Nosso estudo vai ao encontro de tais achados, visto que os afásicos também apresentaram aumento da latência da onda P300 em decorrência da lesão e/ou da idade. 
Diante do que foi observado, percebemos a extrema importância que a avaliação do processamento auditivo possui nessa população. Avaliar para poder estimular e tratar essas habilidades pode proporcionar mais evolução nos sujeitos com afasia de expressão. $\mathrm{O}$ fato de eles possuírem avaliações comportamentais piores do que as eletrofisiológicas demonstra a necessidade de intervenção terapêutica e treinamento auditivo, pois, com a integridade fisiológica da via, há mais chances de evolução terapêutica e melhoras comportamentais auditivas que podem refletir em benefício para os processos de linguagem.

\title{
5 Conclusão
}

Foi possível avaliar as habilidades do processamento auditivo na maioria dos sujeitos com afasia de expressão de modo comportamental e eletrofisiológico. Não foi observada correlação entre ambas as avaliações, demonstrando que as avaliações comportamentais foram piores do que as eletrofisiológicas.

Portanto, nosso estudo evidencia a importância da avaliação audiológica periférica e central no processo de reabilitação de sujeitos com afasia de expressão.

\section{AUDITORY INFORMATION PROCESSING IN INDIVIDUALS WITH APHASIA}

\begin{abstract}
Introduction: Different types of change in the Central Nervous System (CNS) can result in many types of language disorders and/or speech. If there is any change in the central level, it is possible that some auditory abilities are altered. Objective: To evaluate the auditory processing abilities of behavioral and electrophysiological so in subjects with aphasia of speech and identify the correlation between assessments. Methodology: The study subjects aged between 29 and 72 years, expression aphasia resulting from brain injury (stroke or traumatic brain injury). They underwent audiometric assessment beyond the behavioral and electrophysiological assessment of auditory processing, which included the following tests: sound localization, sequential memory for verbal and nonverbal sounds, default frequency and duration, dichotic digits, compressed speech, identification of synthetic and non-verbal dichotic sentences, plus Auditory Evoked Potential Long Latency P300. Results: All subjects evaluated had abnormalities in the
\end{abstract}


majority of behavioral auditory processing skills. In LLAEP, all showed

potential exogenous (N1, P2) within the expected latency. Only one did not have the potential P300 bilaterally. The other five aphasic showed P300 bilaterally. Conclusion: It was possible to assess auditory processing abilities in nearly all subjects with aphasia expression of behavioral and electrophysiological mode. No significant correlation was observed between assessments, identifying the behavioral assessments were worse than electrophysiologic.

keywords

Aphasia. Auditory Perception. Hearing. Audiology. Evoked Potentials.

referências

ALVARENGA, Kátia de Freitas et al. Potencial cognitivo P300 em indivíduos com diabetes mellitus. Revista Brasileira de Otorrinolaringologia, São Paulo, v. 71,n. 2,p. 202-207, mar./abr. 2005.

Estudo eletrofisiológico do sistema auditivo periférico e central em indivíduos afásicos. Arquivos de Neuro-Psiquiatria, São Paulo, v. 63, n. 1, p. 104-109, mar. 2005.

AMERICAN SPEECH-LANGUAGE-HEARING ASSOCIATION. (Central) Auditory Processing Disorders. Technical Report. 2005. Disponível em: <http://www.asha.org/policy/ TR2005-00043> Acesso em: 24 abr. 2015.

BELLIS, Teri James. Assessment and Management of Central Auditory Processing Disorders in the Educational Setting: From Science to Practice. 2. ed. San Diego: Plural Publishing, 2011.

DIAS, Karin Ziliotto. Processamento auditivo em individuos com síndrome de Asperger. 2005. 383 f. Tese (Doutorado em Ciências) - Escola Paulista de Medicina, Universidade Federal de São Paulo, São Paulo, 2005.

HALL III, James W.; CHANDLER, David. Timpanometria na Audiologia Clínica. In: KATZ, Jack. Tratado de Audiologia Clínica. 4. ed. São Paulo: Manole, 1999. cap. 20, p. 281-297.

HUSAIN, Fatima T.; HORWITZ, Barry. Experimental-neuromodeling framework for understanding auditory object processing: integrating data across multiple scales. Journal of Physiology, Paris, v. 100, n. 1-3, p. 133-141, July/Sept. 2006.

JERGER, Susan. Validation of the pediatric speech intelligibility test in children with central nervous system lesions. Audiology, Basel, v. 26, n. 5, p. 298-311, Feb. 1987.

KIMURA, Doreen. Some effects of temporal-lobe damage on auditory perception. Canadian Journal of Psychology, Toronto, v. 15, n. 3, p. 156-165, 1961.

LLOYD, Lyle L.; KAPLAN, Harriet. Audiometric interpretation: a manual of basic audiometry. Baltimore: University Park Press, 1978. p. 16-17.

McPHERSON, David L. Late potentials of the auditory system. San Diego: Singular Publishing Group, 1996. 147 p.

MARANGONI, Andréa Tortosa et al. Avaliação Eletrofisiológica da Audição em indivíduos após Traumatismo Cranioencefálico. Revista CEFAC, São Paulo, v. 15, n. 1, p. 58-68, jan./fev. 2013. 
MASSI, Giselle A et al. Práticas de letramento no processo de envelhecimento. Revista Brasileira de Geriatria e Gerontologia, Rio de Janeiro, v. 13, n. 1, p. 59-71, jan./abr. 2010.

MELO, Iracema Hermes Pires de et al. Potenciais evocados audotivos de longa latências: um estudo de caso de afasia de expressão. Revista CEFAC, São Paulo, v. 9, n. 3, p. 4171-2126, jul./set. 2007.

MUSIEK, FrankE.; PINHEIRO, Marilyn L.; WILSON, Donald H. Auditory pattern perception in 'split brain' patients. Archives of Otolaryngology, Chicago, v. 106, n. 10, p. 610-612, Oct. 1980

OLIVEIRA, Juliana Casseb; MURPHY, Cristina Ferraz Borges; SCHOCHAT, Eliane. Auditory processing in children with dyslexia: electrophysiological and behavior evaluation. CoDAS, São Paulo, v. 25, n. 1, p. 39-44, 2013

ORTIZ, Karin Zazo; PERONI, Caroline. Compreensão de fala em situação de mensagem competitiva em afásicos. Revista CEFAC, São Paulo, v. 10, n. 2, p. 226-232, abr./ jun. 2008.

PEREIRA, Liliane Desgualdo; SCHOCHAT, Eliane. Testes Auditivos Comportamentais para Avaliação do Processamento Auditivo Central. Barueri: Pró-Fono, 2011. 82 p.

PICTON, Terry W.; HILLYARD, Steven A. Human auditory evoked potentials. II. Effects of attention. Electroencephalography and Clinical Neurophysiology, Amsterdam, v. 36, n. 2, p. 191-199, Feb. 1974

PINEROLI, José C. A. et al. Avaliação auditiva central com BERA e P300 na Doença de Parkinson. Revista Brasileira de Otorrinolaringologia, São Paulo, v. 68, n. 3, 462-466, maio/jun. 2002.

SANTOS, Renata Beatriz Fernandes et al. Avaliação comportamental do processamento auditivo em indivíduos pós-traumatismo cranioencefálico: estudo piloto. Revista CEFAC, São Paulo, v. 15, n. 5, p. 1156-1162, set./out. 2013.

SUTTON, Samuel et al. Evoked potential correlates of stimulus uncertainty. Science, Washington, D.C., v. 150, n. 3700, p. 1187-1188, nov. 1965.

TALARICO, Thais Rodrigues; VENEGAS, Mayra Jacuviske; ORTIZ, Karin Zazo. Perfil populacional de pacientes com distúrbios da comunicação humana decorrentes de lesão cerebral, assistidos em hospital terciário. Revista CEFAC, São Paulo, v. 13, n. 2, p. 330-339, mar./abr. 2011

Data de submissão: 20/11/2014

Data de aprovação: 27/07/2015 